\title{
Investigating Dislocation-Twin Boundary Interactions in Nickel using Diffraction Contrast Scanning Transmission Electron Microscopy
}

\author{
D.L. Medlin ${ }^{1}$, M.L. Bowers ${ }^{2}$, C. Ophus ${ }^{2}$, S.K. Lawrence ${ }^{1}$, B. Somerday ${ }^{1}$, and R.A. Karnesky ${ }^{1}$ \\ ${ }^{1}$ Sandia National Laboratories, Livermore, CA USA \\ ${ }^{2}$ National Center for Electron Microscopy, Molecular Foundry, Lawrence Berkeley National Laboratory, \\ Berkeley CA USA.
}

The interaction of dislocations with and near grain boundaries plays an important role in the strain response of metals. Whether a dislocation is blocked or transmitted at a grain boundary is dependent on a number of inter-related factors including the alignment of slip systems, the inclination of the interface, and the nature of the defect transition states within the interface itself. In this presentation we consider the morphology and character of dislocations interacting with $\{111\}$ and $\{112\}$ twin boundary facets in Ni. Our work is motivated in part by interest in explaining the influence of twin boundaries on the fracture behavior of metals exposed to hydrogen environments. For instance, Bechtle et al. have reported a reduced susceptibility to intergranular hydrogen embrittlement in Ni processed to have a high fraction of $\Sigma 3$ twins and related $\Sigma 3^{\mathrm{n}}$ special boundaries [1]. However, analyses have also shown that although they are resistant to crack propagation, twins can still be sites of preferential crack initiation during loading in the presence of hydrogen [2], raising questions regarding the precise mechanisms of strain localization at such interfaces and how these are changed with exposure to hydrogen.

To address these questions, we are investigating dislocation/twin interactions using Diffraction Contrast Scanning Transmission Electron Microscopy (DC-STEM) [3]. Figure 1(a) shows a dark-field DCSTEM image of dislocations in the vicinity of a $\Sigma 3$ twin in Ni, which has formed $\{111\}$ and $\{112\}$ facets. This material was precharged with $4000 \mathrm{appm} \mathrm{H}_{2}$ and then deformed at room temperature to $3.5 \%$ strain. The higher magnification image in Figure 1(b) shows a network of grain boundary dislocations that have formed on one of the twin boundary facets. We are also conducting tilt series so that we can reconstruct the complex three-dimensional topology of the dislocations both within and in the vicinity of the twin boundaries. Figure 2 shows one such example from a series tilting about a $\Sigma 3$ $\{112\}$ facet. As we will discuss, the configuration of these defect networks can be understood in terms of the underpinning interfacial bicrystallography, which predicts the presence of Burgers vectors of type $(1 / 6)<112>$ and $(1 / 3)<111>$.

References:

[1] S. Bechtle, M. Kumar, B.P. Somerday, M.E. Launey, R.O. Ritchie, Acta Mat. 57 (2009) 4148.

[2] M. Seita, J.P. Hanson, S. Gradečak, M.J. Demkowicz, Nature Communications 6 (2015) 6164.

[3] P.J. Phillips, M.C. Brandes, M.J. Mills, M. De Graef, Ultramicroscopy 111 (2011) 1483-1487.

[4] Sandia National Laboratories is a multi-program laboratory managed and operated by Sandia Corporation, a wholly owned subsidiary of Lockheed Martin Corporation, for the U.S. Department of Energy's National Nuclear Security Administration under contract DE-AC04-94AL85000. DM, SL, BS, and RK acknowledge support from Sandia National Laboratories' Laboratory Directed Research and Development (LDRD) Program. Work at the Molecular Foundry, National Center for Electron Microscopy, was supported by the Office of Science, Office of Basic Energy Sciences, of the U.S. Department of Energy under Contract No. DE-AC02-05CH11231. 

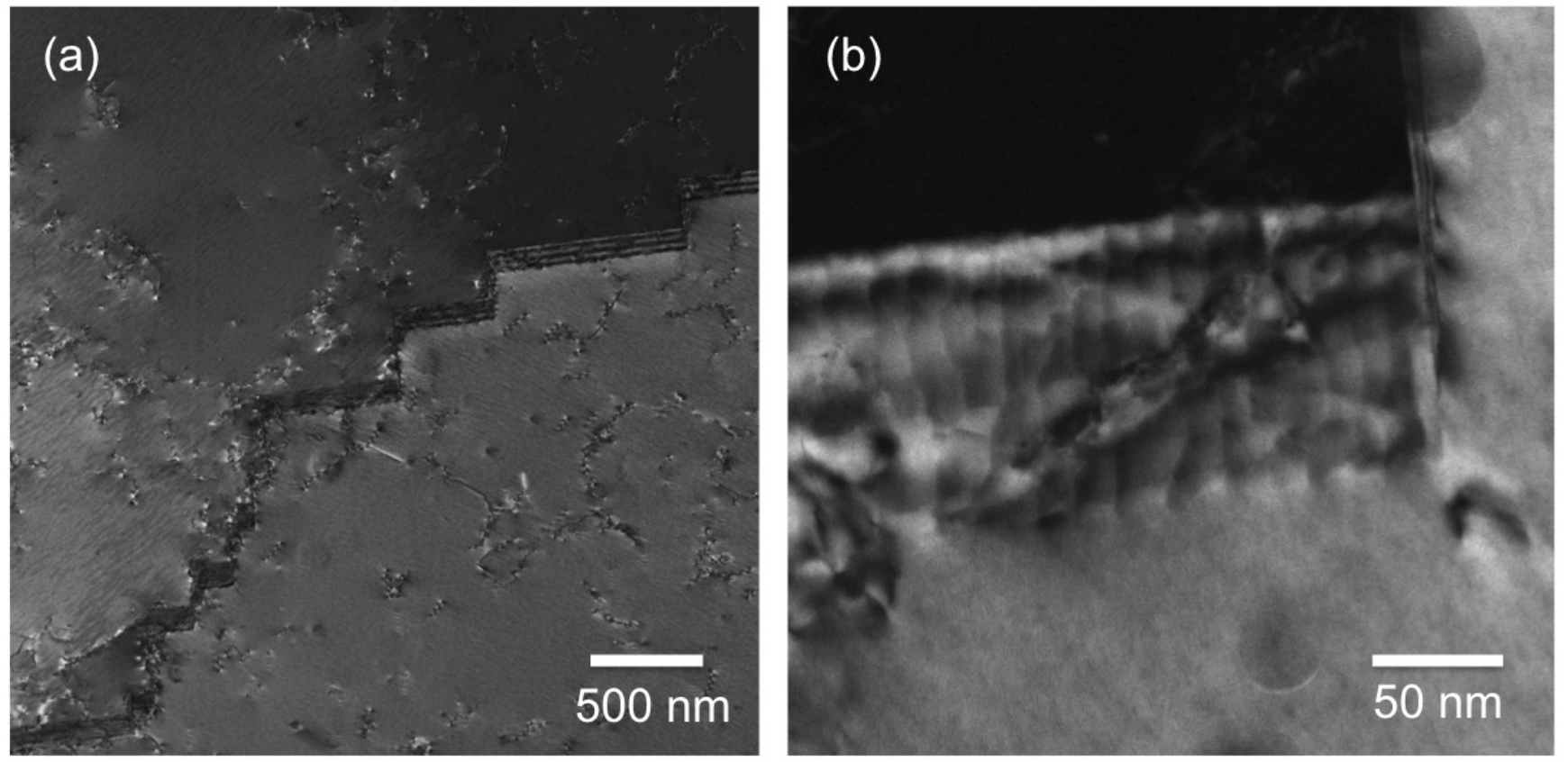

Figure 1. (a) DC-STEM image showing overview of dislocations in vicinity of $\Sigma 3$ boundary in $\mathrm{Ni}$ (4000 appm $\mathrm{H}_{2}$ pre-charged, 3.5\% strain at room temperature). The twin is faceted on $\{111\}$ and $\{112\}$. (b) Detail showing dense network of grain boundary dislocations on a $\{111\}$ twin facet.
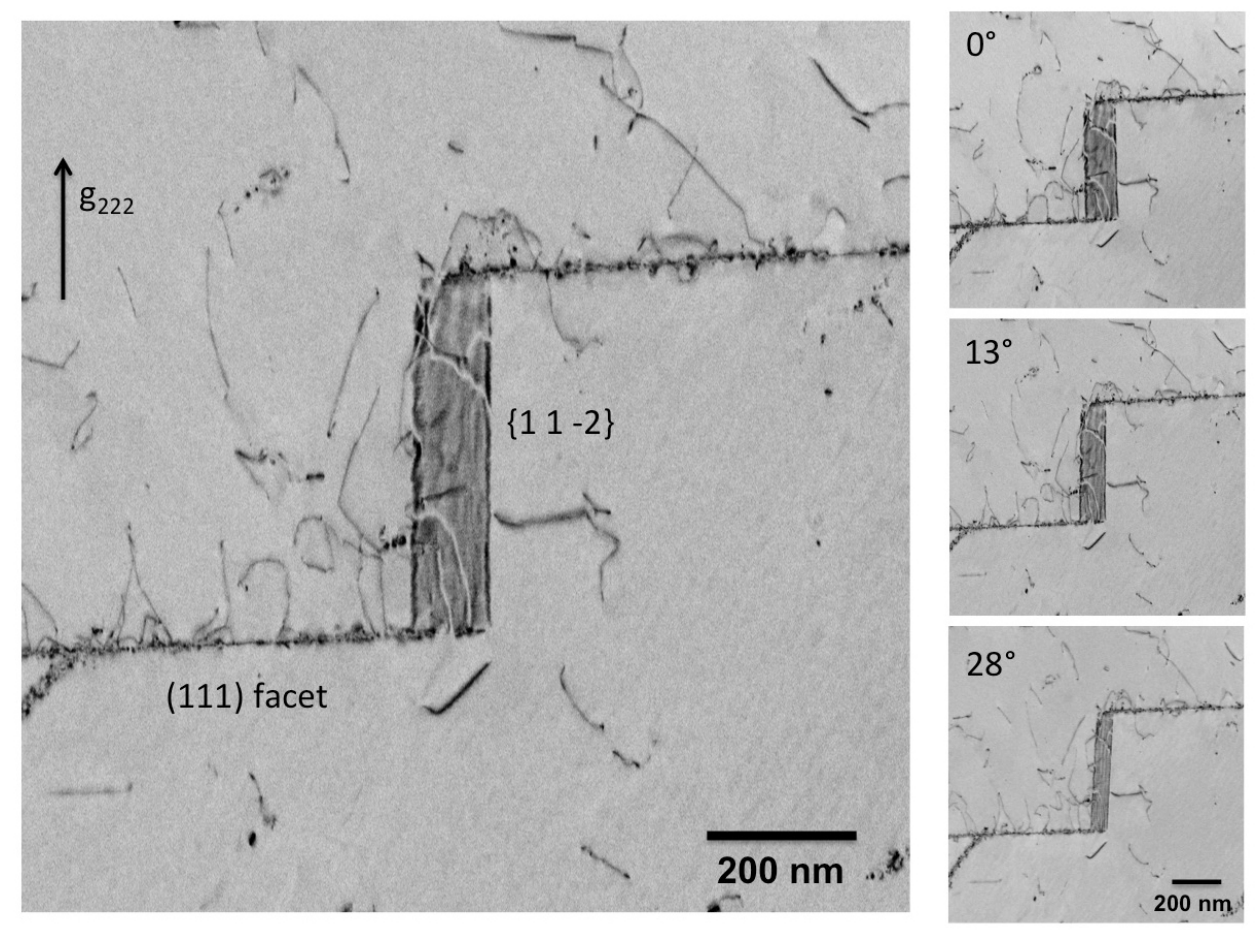

Figure 2. DC-STEM image of dislocations at $\{111\}$ and $\{112\}$ twin boundary facets in Ni. (Dark field image with image contrast reversed for clarity). The insets show frames from a tomographic tilt series. 